\title{
Unsupervised Scoring for Scalable Internet-Based Collaborative Teleoperation
}

\author{
Ken Goldberg, Dezhen Song, In Yong Song, \\ Jane McGonigal, Wei Zheng, UC Berkeley \\ Dana Plautz, Intel Research Lab, Portland
}

\begin{abstract}
For applications in education and entertainment, Scalable Internet-based Collaborative Teleoperation allows many users simultaneously to share control of a single device. Automated numerical methods that can assess and record performance provide an incentive for users to participate and a means to evaluate individual and group performance. In this paper we describe "unsupervised scoring": a numerical approach to assessment based on clustering and response time. Like unsupervised learning, this approach is based on identifying regularities in the input rather than comparing input with desired output specified by an external supervisor. We present an algorithm for rapidly computing user scores that scales linearly with the number of users. We describe an implemented Java-base user interface incorporating this metric, an application based on the classic Twister game, and results where individual scores are compared with group performance.
\end{abstract}

\section{INTRODUCTION}

Remote-controlled machines and teleoperated robots have a long history [30]. Networks such as the Internet provide lowcost and widely-available interfaces that makes such resources accessible to the public. In almost all existing teleoperation systems, a single human remotely controls a single machine. For applications in education and entertainment, we are developing consider Collaborative teleoperation (CT) systems that allow many users to simultaneously share control. In a taxonomy proposed by Tanie et al. [3], these are Multiple Operator Single Robot (MOSR) systems in contrast to conventional Single Operator Single Robot (SOSR) systems.

In MOSR systems, inputs from many participants are combined to generate a single control stream. There can be benefits to collaboration: teamwork is a key element in education at all levels [27], [5], [4] and the group may be more reliable than a single (possibly malicious) participant [11]. In [13], we introduced the "Tele-Actor", a system involving a skilled human with cameras and microphones connected to a wireless digital network, who moves through the remote environment based on live feedback from online users.

In Tele-Actor field tests with students ranging from middle and high-school to graduate students, we discovered that students would often become passive, simply watching the video without participating. Also, instructors asked for a mechanism to quantify student performance for grading purposes. We realized we could address both issues with an "unsupervised

This work was supported in part by the National Science Foundation under IIS-0113147, by the Intel Research Council, and by UC Berkeley's Center for Information Technology Research in the Interest of Society (CITRIS). For more information please contact goldberg@ieor.berkeley.edu.

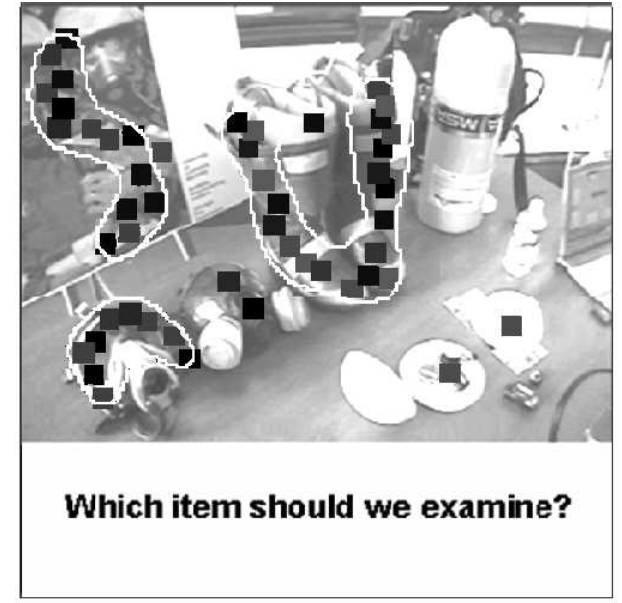

(a)

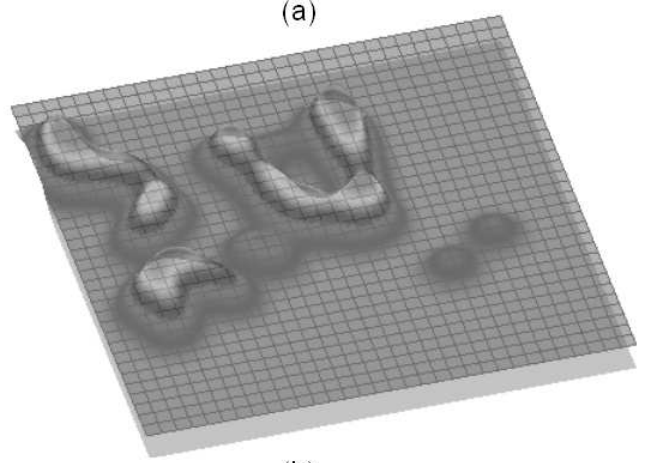

(b)

Fig. 1. Above, (a) illustrates a sample view from our scalable user interface, where spatial inputs from users are represented with colored square markers, in this case indicating points of interest in a live video image. The unsupervised scoring metric assigns a scalar value to each input. Below, (b) illustrates the aggregate (consensus) density distribution that is used to automatically compute scores based on vote arrival time.

scoring" system that provides a competitive element to the interactions and continually assesses students.

This paper describes the unsupervised scoring metric and algorithms for rapidly computing it. In "supervised learning," system outputs are compared against desired outputs and parameters adjusted accordingly. In "unsupervised learning", desired outputs are unspecified: learning proceeds by identifying and rewarding inherent structure in the input patterns. Analogously, "unsupervised scoring" is based on identifying regularities in collective user input rather than comparing these inputs with desired outputs as specified by an external supervisor. 
In our model, individual performance is based on "leadership": how quickly a user anticipates the decision of the majority. As illustrated in Figure 1, the unsupervised scoring metric is based on clustering of user inputs. For $n$ users, computing scores runs in $O(n)$ time. This paper presents problem formulation, distributed algorithms, and experiment results.

\section{RELATED WORK}

Networked robots, controllable over networks such as the Internet, are an active research area. In addition to the challenges associated with time delay, supervisory control, and stability, online robots must be designed to be operated by nonspecialists through intuitive user interfaces and to be accessible 24 hours a day. There are now dozens of Internet robots online, a book from MIT Press [12], and an IEEE Technical Committee on Internet and Online Robots. See [16], [28], [17], [18], [25], [14], [26], [20], [22] examples of recent projects.

Tanie, Matsuhira, Chong, et al. [3] proposed a useful taxonomy for teleoperation systems: Single Operator Single Robot (SOSR), Single Operator Multiple Robot (SOMR), and Multiple Operator Multiple Robot (MOMR).

Most networked robots are SOSR, where control is limited to one human operator at a time. Tanie et al. analyzed an MOMR system where each operator controls one robot arm and the robot arms have overlapping workspaces. They show that predictive displays and scaled rate control are effective in reducing pick-and-place task completion times that require cooperation from multiple arms [3].

In an MOMR project by Elhajj, Fukuda, Liu, Xi, and colleagues [7], two remote human operators collaborate to achieve a shared goal such as maintaining a given force on an object held at one end by a mobile robot and by a multijointed robot at the other. MOMR models are also relevant to online collaborative games such as Quake and The Sims Online, where players remotely control individual avatars in a shared virtual environment.

One precedent for an online MOSR system is described in McDonald, Cannon and colleagues [24]. For waste cleanup, several users to assist remotely using Point-and-Direct (PAD) commands [2]. Users point to cleanup locations in a shared image and a robot excavates each location in turn. In this Internetbased MOSR system, collaboration is serial but pipelined, with overlapping plan and execution phases. The authors demonstrate that such collaboration improves overall execution time but do not address conflict resolution between users.

In [11], Goldberg, Chen, et al present an Internet-based MOSR system that averaged multiple vector inputs to control the position of an industrial robot arm. In [31], we present another MOSR system that allow a group of users to simultaneously share control of a single robotic camera. We formulate MOSR problem as resource allocation problem and develop algorithms for camera control.

Outside of robotics, the notion of MOSR is related to a very broad range of group activities including compute gaming, education, social psychology, voting, etc. Although there currently are no standard "best practices" for the design of scoring systems in the computer games industry, some tentative efforts recently have been made to identify the key features of an effective scoring and ranking model. GamaSutra, the flagship professional resource for digital game designers from around the world, has proposed four characteristics of a successful scoring system. It should be reproducible (consistent), comprehensive (incorporating all significant aspects of game play), sufficiently detailed (so that players understand how it is calculated), and well-balanced (allowing for players to stay competitive even when playing from a disadvantage) [19]. Kriemeier suggests that a failure to address each of these four principles will negatively affect user participation and motivation. Proposed customizations to further increase player motivation in a system with these four standard features include introducing non-zero sum (cooperative) scoring elements and a score decay algorithm that will penalize players for a lower rate of participation in the game. The Tele-Actor unsupervised scoring model aims to incorporate these features, and to investigate their value and effectiveness from a userperspective.

Two different areas in educational psychology investigate the usefulness of games in learning. The first addresses the element of "fun" in games as a powerful motivational tool [10], [15]. In particular, cooperative play models are thought to provide particularly powerful evidence of games as tools of engagement; as Sutton-Smith notes [32], children and young adults are so motivated to be accepted in such play, they make sacrifices of egocentricity for membership in the group, a claim that may be tested by the unsupervised scoring model.

Csikszentmihalyi's theory of "flow" [6], a confidencebuilding state in which a participant becomes absorbed in and highly effective at a particular activity, argues for the importance of feedback as the primary criterion for achieving flow. According to Csikszentmihalyi, flow creates a sense of motivation that is intrinsic to the activity, rather than relying on extrinsic rewards (prizes, grades, acclaim), and intrinsic motivation is ultimately more important for long-term success in any activity. For example: Gee [9] argues that students today are used to experiencing flow and achieving a sense of mastery in their at-home game play, but not in school. Many educators are considering adopting more game-like scenarios for learning as a way to incorporate students' proclivity for structured play.

Group decision-making is sometimes modelled as an nperson (multi-player) cooperative game [33], [1]. We can view the our system as a non-zero sum multi-player game in which users both compete and cooperate to increase their individual leadership scores. Our leadership metric may be useful for evaluating cooperative behavior in real-life [23], [29].

A related question is, how do players learn game strategies and adapt their game play behavior accordingly over time? The study of adaptive learning models in games attempts to develop a theory of "game cognition" that explains why people do not always discover or follow optimal strategies in game play [21]. Erev and Roth [8], for instance, argue for the importance of the presence or absence of reinforcement and feedback within a repetitive game structure as the most important factor in predicting player action. By providing feedback in the form of a leadership score and simultaneously 


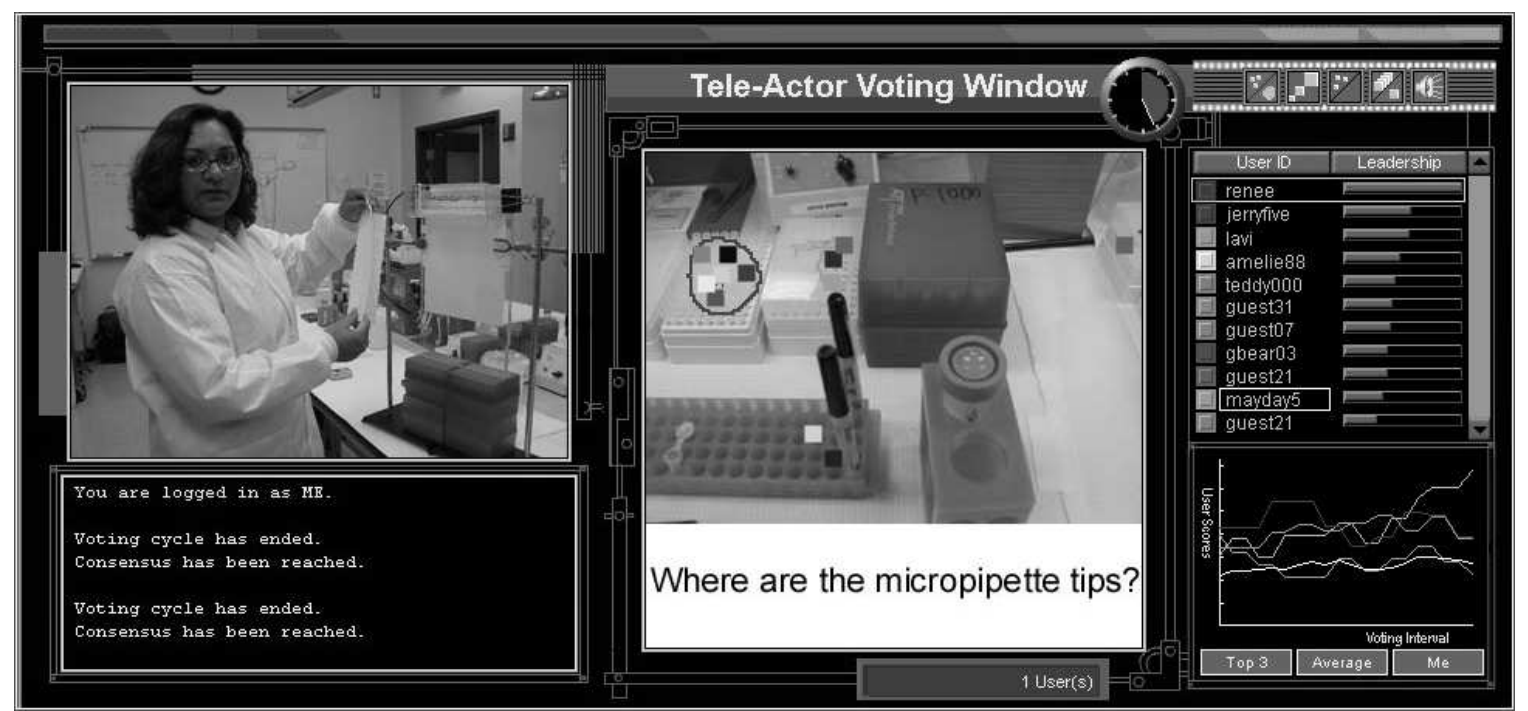

Fig. 2. The Spatial Dynamic Voting (SDV) interface as viewed in the browser by each online voter. Low framerate live video (approx 1 fps) is displayed in the left window. Users vote on spatial preferences using their mouse to position a small marker (votel) in the middle (voting) window, over either a prestored or live image. Users view the position of all votels and can change their votel positions based on group dynamics. As described below, votel positions are processed to assign scores to each user. The list of active voters, ranked by score is displayed in the right window. The lower right window displays a plot of voter score, overall average, and scores for the top three voters.

tracking changes in scores over time, we can numerically compare individual and group performance.

\section{PRoblem Definition}

Figure 2 illustrates the user interface.

In this section, we propose an unsupervised scoring metric based spatial distributions of votes.

\section{A. Inputs and assumptions}

Consider the $k$ th voting image. The server receives a response from user $i$ in the form of an $(x, y)$ mouseclick on image $k$ at time $t$. We define the corresponding votel: $v_{i k}(t)=\left[x_{i k}(t), y_{i k}(t)\right]$.

Each votel represents a user's response ("vote") to the voting image. We model such responses with a voter interest function, a density function based on the bivariate normal distribution:

$$
f_{i k}(x, y) \sim N\left(v_{i k}(t), \Sigma_{i k}(t)\right)
$$

where $v_{i k}(t)$ is the mean vector and $\Sigma_{i k}(t)$ is a $2 \times 2$ variance matrix, such that,

$$
\iint_{\sigma} f_{i k}(x, y) d x d y=1
$$

where $\sigma$ is the area of the voting image. Since $\sigma$ is a bounded $2 \mathrm{D}$ region, the voter interest function is a truncated bivariate normal density function with mean at $v_{i k}(t)$ as illustrated in Figure 3(a).

1) Majority Interest Function: When voting on image $k$ ends at stopping time $T$, the last votel received from each of $n$ active voters determines $V_{k}$, a set of $n$ votels. We define the ensemble interest function for voting image $k$ as the normalized sum of these voter interest functions.

$$
f_{k}(x, y)=\frac{1}{n} \sum_{i=1}^{n} f_{i k}(x, y) \text {. }
$$

2) Consensus Regions: As illustrated in figure 3(c), we can extract spatial regions by cutting the ensemble interest function using a horizontal plane at a height proportional to the overall volume. Let $z_{k}$ be the cutting threshold. The $z_{k}$ value satisfies the condition that the ratio between the partial volume of the ensemble interest function above the horizontal plane and the total volume of the ensemble interest function is constant $r$. We use a value of 0.10 (10\% of the volume lies above the plane). The cutting plane defines an iso-density contour in the ensemble interest function that defines a set of one or more closed subsets of the voting image,

$$
S_{k}=\left\{(x, y) \mid f_{k}(x, y) \geq z_{k}\right\} .
$$

As illustrated in Figure 3(c), we refer to these subsets as consensus regions:

$$
S_{k}=\left\{C_{1 k}, C_{2 k}, \ldots, C_{l k}\right\} .
$$

Since there are $n$ voters, $l \leq n$ is number of consensus regions.

3) Majority Consensus Region: Given $S_{k}, V_{k}$, the majority consensus region is the region with the most votels (breaking ties arbitrarily). Let

$$
I_{k}(i, j)= \begin{cases}1 & \text { if }\left[x_{i k}(T), y_{i k}(T)\right] \in C_{j k} \\ 0 & \text { otherwise }\end{cases}
$$

The count

$$
n_{k j}=\sum_{i=1}^{n} I_{k}(i, j)
$$

is the number of votels inside consensus region $j$ of voting image $k$. Breaking ties arbitrarily, let $C_{k}^{*}$, the majority consensus region, be any $C_{j k}$ with $\max n_{k j}$.

\section{B. Unsupervised Scoring Metric}

We measure individual performance in terms of "leadership". By definition, a "leader" anticipates the choices of the 


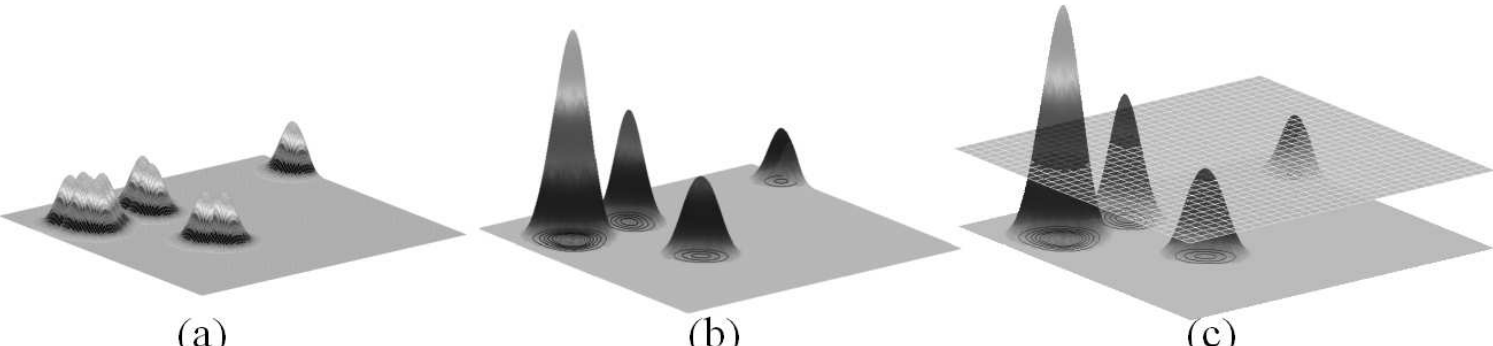

(a)

(b)

(c)

Fig. 3. An example of voter interest functions, the corresponding majority interest function, and an illustration of consensus region generation for the voting image in Figure 2.

group. In our context, a leader is an individual who votes early in a position consistent with the majority consensus region. Define $I_{s}$ is an outcome index for voter $i$ and voting image $s$ :

$$
I_{s, i}= \begin{cases}1 & \text { if }\left[x_{i, s}\left(T_{s}\right), y_{i, s}\left(T_{s}\right)\right] \in C_{s}^{*} \\ 0 & \text { otherwise }\end{cases}
$$

Define $t_{s, i}$ as the duration of the time that voter $i$ 's votel stays in the majority interest region for the $s^{t h}$ voting image, $T_{s}$ is the total voting time for voting image $s$. Therefore term $\frac{T_{s}-t_{s, i}}{T_{s}} I_{s, i}$ characterizes how well the voter anticipated the majority consensus region for the voting image $s$.

To smooth out rapid changes in user scores, we pass the term to the following low pass filter to get a stabilized "Leadership score":

$$
L_{k+1, i}=(1-\alpha) L_{k, i}+\alpha \frac{T_{k}-t_{k, i}}{T_{k}} I_{k, i}
$$

where the initial value $L_{0, i}=0$ for each voter $i$. The value of filter factor $\alpha$ is set to 0.1 in our experiments to allow smooth fluctuation in user scores.

Note that this scoring metric depends only on the spatiotemporal pattern of votes and does not require a human expert.

\section{Distributed Algorithm}

To compute the unsupervised score for each user, we start by maintaining the ensemble interest function with a grid. We partition the voting image into $160 \times 160$ regular cells. For each voter interest function $f_{i k}(x, y)$, we discretize it into a 2D array with respect to the same lattice resolution. Depending on the variance of the Gaussian function and accuracy threshold, each element of the 2D array only contains constant number of non-zero entries. Therefore, to compute the ensemble interest function for $\mathrm{n}$ votes, we add those $\mathrm{n} 2 \mathrm{D}$ arrays into the cells. This operation takes $O(n)$. Figure 3(b) shows the shape of the ensemble interest function for the voter interest function in 3(a).

As illustrated in figure 3(c), we can extract spatial regions by cutting the ensemble interest function using a horizontal cutting plane. The next step is to compute the height of the cutting plane $z_{k}$ for the given volume ratio $r$. Define $r(z)$, $z>0$ be the volume ratio between the partial volume of the majority interest function above the cutting plane with height $z$ and the total volume of the ensemble interest function. As $z$ increases, the horizontal plane rises. Hence $r(z)$ is a monotonic decreasing function of $z$. A binary search can find $z_{k}$ in $O(\log (1 / \varepsilon))$ steps with error $\varepsilon$. Since we need to compute the partial volume for each step, which takes at most $O(m)$ for $m$ cells in the grid. Therefore, the complexity for computing the threshold is $O(\log (1 / \varepsilon) m)$. If we consider $\varepsilon$ as a constant, then it is $O(m)$.

The second step is to perform the threshold for the ensemble function and find connected components, each of which forms a consensus region. The connected components algorithm makes two passes through the 2D array processing a row of cells at a time, from left to right. During the first pass, each cell is assigned the minimum label of its neighbors or if none exists, a new label is assigned. If neighboring cells have different labels, those labels are considered equivalent and entered into an equivalence table. The second pass uses the equivalence table to assign the minimum equivalent label to each non-zero entry. Since, this step also takes $O(m)$ time, the total computation time for computing the consensus regions for a given ensemble interest function is $O(m)$.

To determine the majority consensus region, we need to count the number of votels inside each consensus region. For this purpose, each cell maintains a votel count that is updated during votel insertions. In a single pass, we can sum the number of votels in the cells belonging to each consensus region. Thus, this step takes $O(m)$ time.

If we add computation time of algorithms for ensemble interest function, consensus regions, majority consensus regions, and leadership score together, the total computation time is $O(n+m)$

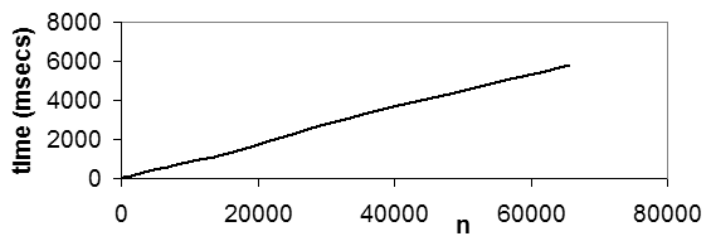

Fig. 4. Processing time for computing the unsupervised scoring metric as a function of the number of voters based on trials using random voter positions.

The algorithm has been implemented and runs on the client side using Java. We tested the algorithm using a $750 \mathrm{Mhz}$ PC Laptop with $256 \mathrm{Mb}$ RAM. Figure 4 illustrates the linear scalability of the algorithm in terms of number of voters. The Tele-Actor server is primarily responsible for distributing voting data to all users. Each client sends the user's voting data and receives updated voting data from the Tele-Actor server 
every 1.0 seconds. For every server update, at most $n$ new voter interest functions will need to be inserted. Using the updated interest functions, the consensus regions and the majority consensus region are recomputed. When a voting cycle ends, each voter computes only the voter's new leadership score, thus distributing the scoring calculations among the clients.

\section{The “Tele-Twister" ApPlicAtion}

To understand how the unsupervised scoring metric works with groups of participants and to test software reliability, we developed a collaborative teleoperation system based on Twister, the classic party game. The result is a sequence of multi-player non-zero sum games embedded inside a sequence of two-player zero sum games.

Twister was the first board game where human bodies are the board pieces. In the classic game, human players interact over a large plastic playing surface. Players sequentially place their hands and feet on colored circular targets chosen randomly (eg, Left foot: GREEN). The challenge for players is to maintain placement of hands and feet in increasingly difficult combinations without falling over.

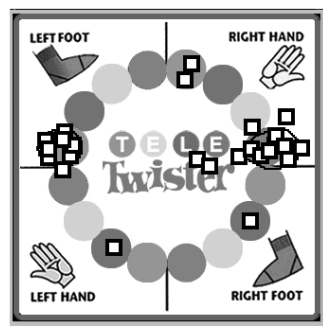

(a)

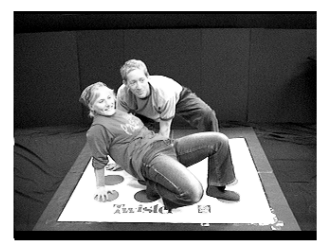

(c)

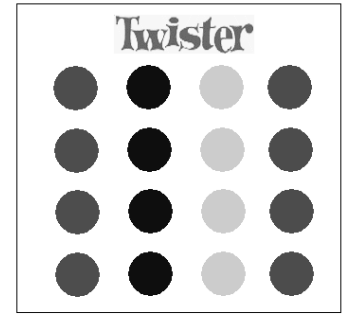

(b)

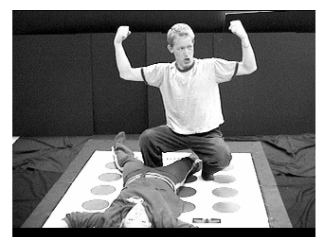

(d)
Fig. 5. Tele-Twister: application of scalable collaborative teleoperation where teams of online players direct the movements of human players in real time.

In our version, Tele-Twister, there are two human players called "twisters". One twister is dressed in red, the other in blue, as illustrated in figure 5. Remote participants ("voters"), download the Java applet and are assigned to one of two teams, red or blue. In Tele-Twister, random target selection is replaced by the teams, who view game status using the low framerate video and vote using the interface to collectively decide on the targets and compete to win: having their opponent fall over first.

A typical voting pattern is illustrated in Figure 5(a). Votel positions for all online voters are updated every second and displayed at each voter's browser. Consensus regions are computed and updated continuously by each browser. voters are free to change their votel position at any time during the voting cycle, but when it ends, the majority cluster determines the next move for the human twisters.
Tele-Twister is thus strategic: the red team chooses targets that are easy for the red twister and difficult for the blue twister, and vice versa for the blue team. Tele-Twister encourages active collaboration within teams and competition between teams. In this context, the unsupervised scoring metric rewards active participation and collaboration (voters who don't vote or are outside the majority region at the end of a voting cycle receive a zero score).

Figure 5(b) shows the board layout, with 16 circles: red, blue, yellow, and green. Since August 12th, 2003 we have conducted public "field tests" on Fridays, from 12-1pm Pacific Time. These field tests attract 10-25 online participants, who vote in alternating 30 second voting periods until one twister falls, ending the round. Typically, each round continues for 10-20 voting periods, so we conduct 4-5 rounds during each field test. Figure 5(c) and (d) are two snapshots from a typical round.

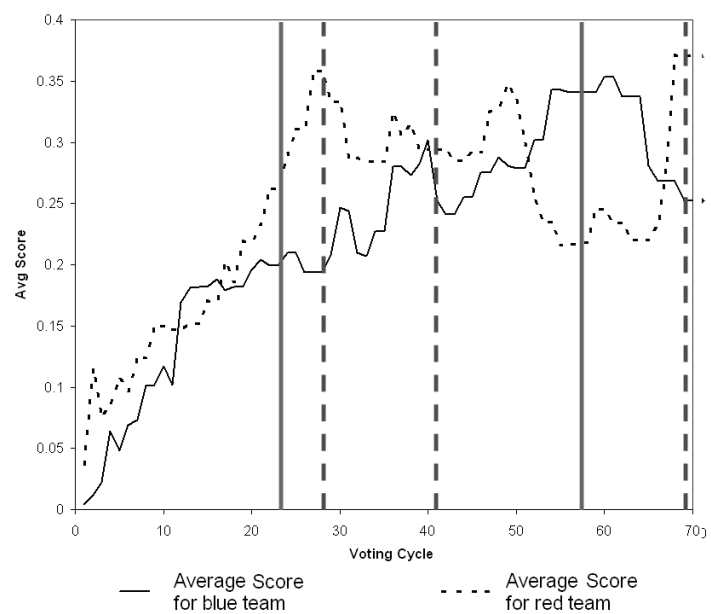

Fig. 6. Plot of unsupervised scoring metric from the Sept 262003 field test with two teams of 21 online voters, for five rounds of the Tele-Twister game. The figure plots average score for members of each team during sequential voting cycles. Vertical bars indicate the end of a round and which team wins. A solid vertical line indicates that the blue team won that round and a dashed vertical line indicates that the red team won.

Figure 6 shows scoring data from the field test on Friday September 26th, 2003. All players begin the field test with a score of zero, so average score per team climbs during the initial voting cycles: the blue team wins the first round after approx 23 voting cycles.

How do user scores correlate with task performance (winning the round)? Note that in the four subsequent rounds, the team with the highest average score consistently wins the round: the red team wins rounds 2,3 and 5 and the blue team wins round 4 . During round 4 , members of the red team had difficulty agreeing on the appropriate next move, reducing the score of many red voters and in turn the average red team score. The lack of consensus (ie. "split votes") resulted in a loss during that round.

A team has higher average scores when the team collaborates, reaching consensus faster. This does not always correlate with success: it can lead to short-term snap decisions that may appear strong but are strategically weak.

Figure 7 plots individual voter scores from the same field 


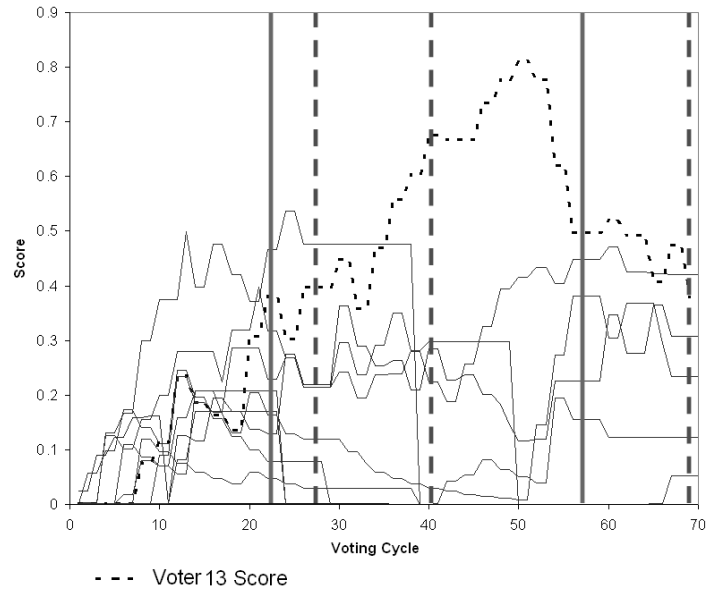

Fig. 7. From the same Sept 262003 field test, plot of unsupervised scoring metric for seven individual voters from the Blue team.

test. Note that the score of voter 13 is consistently higher. In this case voter 13 is a member of our lab who has played the game during many previous rounds and has developed skill at picking the next moves. Other players follow his moves, resulting in a high score.

The Tele-Twister interface is available online at: http://www.tele-actor.net/tele-twister/

\section{FUTURE WORK}

This paper describes an unsupervised scoring metric for collaborative teleoperation that encourages active participation and collaboration, and a scalable distributed algorithm for automatically computing it. To understand how the scoring metric works with groups of participants and to test software reliability, we developed a collaborative teleoperation system based on a sequence of multi-player non-zero sum games embedded inside a sequence of two-player zero sum games. Initial results suggest that the metric encourages active participation and correlates reasonably with task performance.

\section{ACKNOWLEDGMENTS}

Thanks to T. Shlain, E. Paulos and D. Pescovitz for valuable input on initial experiments, the students who have participated in the project: J. Wang, K. "Gopal" Gopalakrishnan, our sponsors the National Science Foundation and Intel Corporation, and to research colleagues: R. Bajcsy, J. Canny, P. Wright, G. Niemeyer, R. Siegwart, D. Greenbaum, and T. Shlain.

\section{REFERENCES}

[1] R.J. Aumann and J.H. Dreze. Cooperative games with coalition structures. International Journal of Game Theory, 3:217-237, 1974.

[2] D. J. Cannon. Point-And-Direct Telerobotics: Object Level Strategic Supervisory Control in Unstructured Interactive Human-Machine System Environments. PhD thesis, Stanford Mechanical Engineering, June 1992.

[3] N. Chong, T. Kotoku, K. Ohba, K. Komoriya, N. Matsuhira, and K. Tanie. Remote coordinated controls in multiple telerobot cooperation. In IEEE International Conference on Robotics and Automation, volume 4, pages 3138-3343, April 2000.

[4] P. Coppin and W. Whittaker et al. Eventscope: Amplifying human knowledge and experience via intelligent robotic systems and information interaction. In 9th IEEE International Workshop on Robot and Human Interactive Communication (Ro-man), 2000.
[5] C. H. Crouch and E. Mazur. Peer instruction: Ten years of experience and results. Americian Journal of Physices, 69(9):970-977, September 2001.

[6] M. Csikszentmihalyi. Beyond Boredom and Anxiety. Jossey-Bass Publishers, 1982.

[7] I. Elhajj, J. Tan, N. Xi, W.K. Fung, Y.H. Liu, T. Kaga, Y. Hasegawa, and T. Fukuda. Multi-site internet-based tele-cooperation. Integrated Computer-Aided Engineering, 9(2):117-27, 2002.

[8] I. Erev and A. E. Roth. Predicting how people play games: Reinforcement learning in experimental games with unique, mixed strategy equilibria. American Economic Review, 88(4):848-81, September 1998.

[9] J. P. Gee. High score education. Wired, 11(5), May 2003.

[10] E. Goffman. Strategic Interaction. University of Pennsylvania Press, 1969.

[11] K. Goldberg and B. Chen. Collaborative control of robot motion: Robustness to error. In International Conference on Intelligent Robots and Systems (IROS), volume 2, pages 655-660, October 2001.

[12] K. Goldberg and R. Siegwart, editors. Beyond Webcams: An Introduction to Online Robots. MIT Press, 2002.

[13] K. Goldberg, D. Song, and A. Levandowski. Collaborative teleoperation using networked spatial dynamic voting. The Proceedings of The IEEE, 91(3):430-439, March 2003.

[14] K. Han, Y. Kim, J. Kim, and S.Hsia. Internet control of personal robot between kaist and uc davis. In IEEE International Conference on Robotics and Automation (ICRA), 2002.

[15] W. Hartmann and B. Rollett. Positive interaction in the elementary school. pages 195-202, 1994.

[16] H. Hu, L. Yu, P. W. Tsui, and Q. Zhou. Internet-based robotic systems for teleoperation. Assemby Automation, 21(2):143-151, May 2001.

[17] S. Jia, Y. Hada, G. Ye, and K. Takase. Distributed telecare robotic systems using corba as a communication architecture. In IEEE International Conference on Robotics and Automation (ICRA), 2002.

[18] J. Kim, B. Choi, S. Park, K.Kim, and S. Ko. Remote control system using real-time mpeg-4 streaming technology for mobile robot. In IEEE International Conference on Consumer Electronics, 2002.

[19] B. Kreimeier. Rising from the ranks:rating for multiplayer games. http://www.gamasutra.com/features/20000209/kreimeier_pfv.htm.

[20] R.C. Luo and T. M. Chen. Development of a multibehavior-based mobile robot for remote supervisory control through the internet. IEEE/ASME Transactions on Mechatronics, 5(4):376-385, 2000.

[21] R. Marimon and E. McGrattan. On adaptive learning in strategic games. 1995

[22] R. Marin, P.J. Sanz, and J.S. Sanchez. Object recognition and incremental learning algorithms for a web-based telerobotic system. In IEEE International Conference on Consumer Electronics, 2002.

[23] A. Mas-Collell. An equivalence theorem for a bargaining set. Journal of Math and Economics, (18):129-139, 1989.

[24] M. McDonald, D. Small, C. Graves, and D. Cannon. Virtual collaborative control to improve intelligent robotic system efficiency and quality. In IEEE International Conference on Robotics and Automation, volume 1, pages 418-424, April 1997.

[25] T. Mirfakhrai and S. Payandeh. A delay prediction approach for teleoperation over the internet. In IEEE International Conference on Robotics and Automation (ICRA), 2002.

[26] L. Ngai, W.S. Newman, and V. Liberatore. An experiment in internetbased, human-assisted robotics. In IEEE International Conference on Robotics and Automation (ICRA), 2002.

[27] B. Rogoff, E. Matusov, and C. White. Models of teaching and learning: Participation in a community of learners. Oxford, England: Blackwell, 1996.

[28] R. Safaric, M. Debevc, R. Parkin, and S. Uran. Telerobotics experiments via internet. IEEE Transactions on Industrial Electronics, 48(2):424-31, April 2001.

[29] P. Shenoy. On coalition formation: A game-theoretical approach. International Journal of Game Theory, 8:133-164, 1979.

[30] Thomas B. Sheridan. Telerobotics, Automation, and Human Supervisory Control. MIT Press, 1992

[31] D. Song and K. Goldberg. Sharecam part I: Interface, system architecture, and implementation of a collaboratively controlled robotic webcam. In IEEE/RSJ International Conference on Intelligent Robots (IROS), Nov. 2003.

[32] B. Sutton-Smith. The Ambiguity of Play. Harvard University Press, 1997.

[33] J. von Neumann and O. Morgenstern. The Theory of Games and Economic Behavior. Princeton University Press, 1953. 